\title{
Cholera in the United States, 2001-2011: a reflection of patterns of global epidemiology and travel
}

\author{
A. LOHARIKAR ${ }^{1 *}$,A.E. NEWTON ${ }^{2}$, S. STROIKA ${ }^{3}$, M. FREEMAN ${ }^{3}$, \\ K. D. GREENE ${ }^{3}$, M. B. PARSONS ${ }^{3}$, C. BOPP ${ }^{3}$, D. TALKINGTON ${ }^{3}$, E. D. MINTZ \\ AND B. E. MAHON ${ }^{2}$ \\ ${ }^{1}$ Office of Workforce and Career Development, Centers for Disease Control and Prevention, Atlanta, GA, USA \\ ${ }^{2}$ Enteric Diseases Epidemiology Branch, Division of Foodborne, Waterborne and Environmental Diseases, \\ Centers for Disease Control and Prevention, Atlanta, GA, USA \\ ${ }^{3}$ Enteric Diseases Laboratory Branch, Division of Foodborne, Waterborne and Environmental Diseases, Centers \\ for Disease Control and Prevention, Atlanta, GA, USA \\ ${ }^{4}$ Waterborne Disease Prevention Branch, Division of Foodborne, Waterborne, and Environmental Diseases, \\ Centers for Disease Control and Prevention, Atlanta, GA, USA
}

Received 14 November 2013; Final revision 29 March 2014; Accepted 22 April 2014;

first published online 28 May 2014

\section{SUMMARY}

US cholera surveillance offers insight into global and domestic trends. Between 2001 and 2011, 111 cases were reported to the Centers for Disease Control and Prevention. Cholera was associated with international travel in $90(81 \%)$ patients and was domestically acquired in $20(18 \%)$ patients; for one patient, information was not available. From January 2001 to October 2010, the $42(47 \%)$ travel-associated cases were associated with travel to Asia. In October 2010, a cholera epidemic started in Haiti, soon spreading to the Dominican Republic (Hispaniola). From then to December $2011,40(83 \%)$ of the 48 travel-associated cases were associated with travel to Hispaniola. Of 20 patients who acquired cholera domestically, 17 (85\%) reported seafood consumption; $10(59 \%)$ ate seafood from the US Gulf Coast. In summary, an increase in travel-associated US cholera cases was associated with epidemic cholera in Hispaniola in 2010-2011. Travel to Asia and consumption of Gulf Coast seafood remained important sources of US cholera cases.

Key words: Cholera, diarrhoeal disease, Haiti, Hispaniola.

\section{INTRODUCTION}

Cholera continues to ravage populations in many developing countries, most recently in Haiti and the Dominican Republic, the two countries that form the island of Hispaniola [1, 2], although cholera transmission and effective measures for its prevention have been understood for over 150 years [3, 4]. For over a century, most cases of cholera identified in the

\footnotetext{
* Author for correspondence: Dr A. Loharikar, 1600 Clifton Rd, Atlanta, GA 30333, USA.

(Email: anagha.loharikar@gmail.com)
}

United States have been associated with travel to countries with endemic cholera [5-9]. Domestically acquired cases are primarily associated with the consumption of raw or undercooked seafood harvested from the US Gulf Coast [10-12]. Periodic reviews of US cholera surveillance offer a window on the global cholera situation; this report summarizes cholera cases diagnosed in the United States during 2001-2011.

\section{METHODS}

Cases of cholera that occur in the United States are reported by state and local health departments to 
the Centers for Disease Control and Prevention (CDC) via the Cholera and Other Vibrio Illness Surveillance (COVIS) system [13]. CDC requests isolates from all suspected cases for confirmatory testing. A confirmed case of cholera is defined as an illness characterized by diarrhea, vomiting, or both with (1) isolation of toxigenic Vibrio cholerae serogroup $\mathrm{O} 1$ or $\mathrm{O} 139$ from stool or vomitus or (2) serological evidence of recent $\mathrm{O} 1$ infection, defined as a vibriocidal antibody titre $\geqslant 640$ in acute or early convalescent phase sera in a person epidemiologically linked to a confirmed cholera case. Data reported to COVIS include demographic, clinical, and exposure information, including domestic and international travel and seafood consumption within 7 days of illness onset. A travel-associated case is defined as cholera in a person who travelled outside the United States during the 7 days before illness onset; cases in persons who report no such travel are considered domestically acquired, and cases in persons for whom information about travel is not available are categorized as unknown. If more than one destination country is reported, exposure is assumed to have occurred where cholera is currently circulating. Cases reported to COVIS with onset from 1 January 2001 to 31 December 2011 were included in this review.

All $V$. cholerae serogroup $\mathrm{O} 1$ and $\mathrm{O} 139$ isolates are confirmed on the basis of agglutination in specific antiserum. Boiled lysates of $V$. cholerae are amplified by polymerase chain reaction (PCR) to detect the presence of cholera toxin (ctxA) [14], biotype-specific (tcp A) genes [15], and species-specific gene sequences (ompW, toxR) [16, 17]. Molecular subtyping by pulsed-field gel electrophoresis (PFGE) is conducted [18]. The resulting PFGE patterns are analysed using BioNumerics software (Applied Maths, USA) and uploaded to the Vibrio cholerae National PulseNet database where comparisons to previously reported $V$. cholerae isolates are conducted. PulseNet is the national molecular subtyping network for foodborne bacterial pathogens. Susceptibility testing of toxigenic $V$. cholerae isolates is performed by the disk diffusion method for the following antimicrobials: kanamycin (included in testing until 2010), amoxicillinclavulanate (included in testing beginning in 2010 for patients who reported travel to Haiti), ampicillin, chloramphenicol, ciprofloxacin, furazolidone, nalidixic acid, streptomycin, sulfisoxazole, trimethoprimsulfamethoxazole and tetracycline, in accordance with Clinical Laboratory Standards Institute (CLSI) recommendations [19, 20]. Escherichia coli ATCC
25922 and Pseudomonas aeruginosa ATCC 27853 were used as internal quality controls. Interpretive criteria specific for $V$. cholerae were applied when available [19], or CLSI criteria for Enterobacteriaceae were used [20].

\section{RESULTS}

\section{Demographic and clinical information}

COVIS received reports of 111 confirmed cases of cholera diagnosed in the United States between 1 January 2001 and 31 December 2011. Cases were reported by 27 states and two territories (Fig. 1) with onset dates ranging from 7 April 2001 to 21 November 2011 (Fig. 2). A marked increase in reports occurred after epidemic cholera began in Haiti in October 2010; 46\% of all cases over the 11 -year study period were reported in the subsequent 14 months.

Fifty-six patients $(50 \%)$ were female. Patients' median age was 44 years (range 1-85 years); nine ( $8 \%$ ) were aged $<10$ years, $95(86 \%)$ were $10-64$ years and $17(15 \%)$ were $\geqslant 65$ years (Table 1$)$. Of the 111 patients, 109 (98\%) reported diarrhoea, and 52 $(47 \%)$ reported vomiting. Other reported symptoms included abdominal cramps (52\%), nausea (46\%), fever $(14 \%)$, muscle aches $(13 \%)$, headache $(11 \%)$, and blood in stool (5\%). Of the 90 patients with available information, $56(62 \%)$ were hospitalized. In hospitalized patients with severe complications, four $(4 \%)$ patients had acute renal failure, two $(2 \%)$ had cardiopulmonary arrest, and one (1\%) had shock; none died. In all, $81(73 \%)$ patients received an antimicrobial agent for treatment.

\section{Exposures (source of illness)}

\section{Travel-associated cases}

In $90(81 \%)$ patients, cholera was travel-associated. Travel to Hispaniola accounted for $40(44 \%)$ cases (Haiti, 29; Dominican Republic, 11), to South Asia for $35(39 \%)$ cases (India, 17; Pakistan, 13; Bangladesh, 4; Nepal, 1), to Southeast Asia for 13 (14\%) cases (Philippines, 8; Indonesia, 3; Thailand, 2); and to West Africa for two (2\%) cases (Ghana, 1; Benin, 1). The 42 travel-associated cases with onset from January 2001 to 21 October 2010 were all associated with travel to South and Southeast Asia. After 21 October 2010, $40(83 \%)$ of the 48 


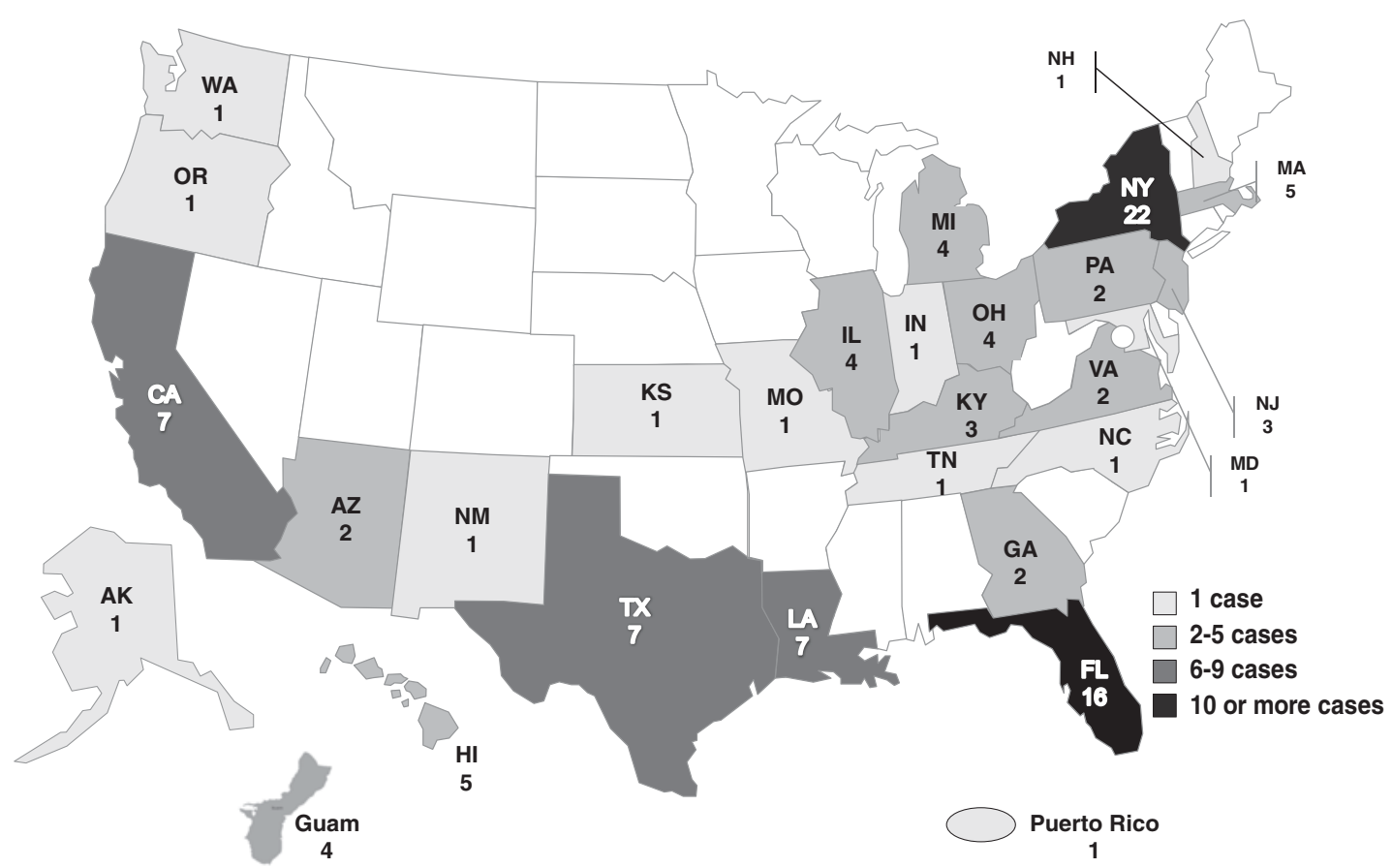

Fig. 1. States and US territories reporting cholera cases, 2001-2011 ( $n=111$ cases).

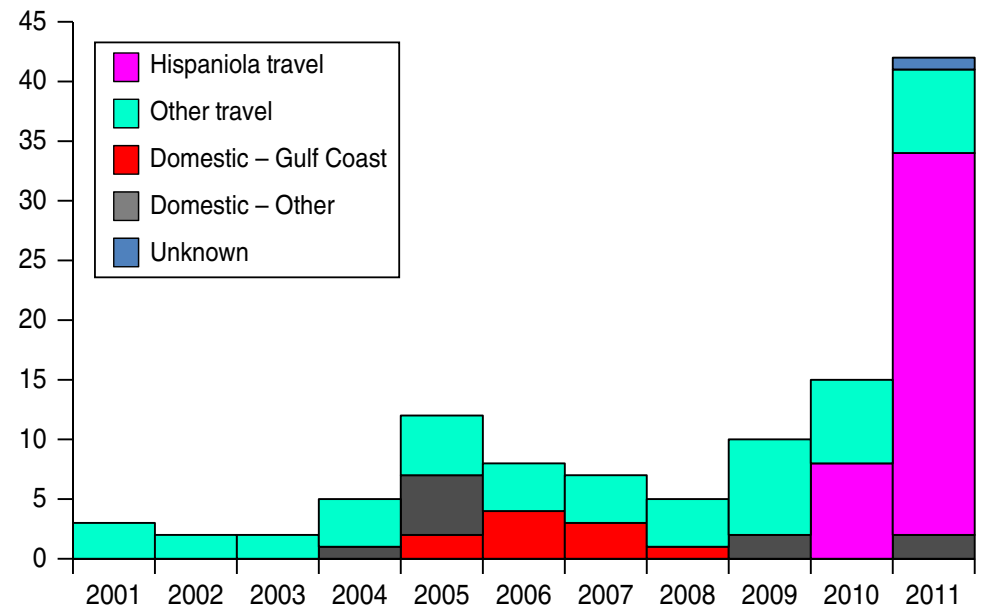

Fig. 2. Number of cholera cases by year, and by source 2001-2011, United States ( $n=111$ cases).

travel-associated cases were associated with travel to Hispaniola.

Travel-associated cases were reported by 27 states. Most of the 40 cases associated with travel to Hispaniola were reported by Florida (14 cases) and New York (12 cases); 10 other states reported the other 14 cases associated with travel to Hispaniola. The 49 cases associated with travel to destinations other than Hispaniola were more widely distributed across the United States, with 22 states reporting cases with no more than eight reports from a single state.

Reported reasons for travel included visiting friends and relatives $(62 \%$, including nine who attended a wedding in the Dominican Republic on 22 January 2011 [21]), tourism (7\%), business (7\%), medical missions or other relief work $(9 \%$, most with travel to Hispaniola), and immigration to the United States $(5 \%)$; information on the reason for travel was not available for $12 \%$. 
Table 1. Cholera cases by age group and source, 20012011, United States ( $n=111$ cases)

\begin{tabular}{lllll}
\hline \hline $\begin{array}{l}\text { Age } \\
\text { group } \\
\text { (years) }\end{array}$ & $\begin{array}{l}\text { Travel- } \\
\text { associated } \\
(n=90)\end{array}$ & $\begin{array}{l}\text { Domestically } \\
\text { acquired } \\
(n=20)\end{array}$ & $\begin{array}{l}\text { Unknown } \\
\text { source } \\
(n=1)\end{array}$ & $\begin{array}{l}\text { Total } \\
(n=111)\end{array}$ \\
\hline$<1$ & 0 & 0 & 0 & 0 \\
1 & 1 & 0 & 0 & 1 \\
$2-4$ & 5 & 1 & 0 & 6 \\
$5-9$ & 2 & 0 & 0 & 2 \\
$10-19$ & 7 & 0 & 0 & 7 \\
$20-29$ & 12 & 2 & 1 & 15 \\
$30-64$ & 50 & 13 & 0 & 63 \\
$\geqslant 65$ & 13 & 4 & 0 & 17 \\
\hline \hline
\end{tabular}

\section{Domestically acquired cases}

Twenty (18\%) domestically acquired cases were reported by seven states and one territory; 19 (95\%) reported seafood consumption. Ten $(50 \%)$ were associated with consumption of Gulf Coast seafood; seven of these patients resided in Louisiana. Gulf Coast seafood consumed included: raw oysters (two cases), boiled and/or raw crabs (eight cases), cooked shrimp (five cases), and fish (three cases). The other $10(50 \%)$ domestically acquired cases included six patients with a history of non-Gulf Coast seafood consumption for whom the source of seafood was not known, three patients with a history of imported seafood consumption (reheated conch imported from Haiti for one, fried shrimp imported from either Nicaragua or Indonesia for one, and raw shrimp imported from Asia for one) and one patient who reported eating no seafood (a young child whose source was unclear but who had contact with travellers from Pakistan). Of the 19 patients with domestically acquired cholera who reported seafood consumption, eight (47\%) consumed the seafood raw.

\section{Unknown source}

One $(1 \%)$ patient was lost to follow-up, so no information about either the location or the likely source of exposure was available.

\section{Laboratory results}

Cholera was confirmed by isolation of toxigenic $V$. cholerae from stool in 108/111 cases; 107 (96\%) stool specimens yielded $V$. cholerae serogroup $\mathrm{O} 1$, all biotype El Tor; 22 (21\%) were serotype Inaba, and $85(79 \%)$ were serotype Ogawa (Table 2). One
(1\%) stool specimen collected in 2009 yielded serogroup O139; the patient had domestically acquired cholera and reported consuming imported raw shrimp purchased from a seafood market specializing in Asian foods. Three cases $(3 \%$, all in patients with a history of travel to Hispaniola) were confirmed serologically.

PFGE results were available for $93 \quad(87 \%)$ $V$. cholerae isolates (Table 2). The PFGE pattern combinations of $45(48 \%)$ serogroup $\mathrm{O} 1$ isolates were indistinguishable from isolates obtained from patients in Hispaniola, [21, 22] labelled the 'Haiti pattern'. Of these 45 patients, $26(60 \%)$ reported travel to Haiti, 10 (22\%) to the Dominican Republic, five (11\%) to India, one to Pakistan $(2 \%)$, one $(2 \%)$ to Benin; two $(4 \%)$ reported no travel but consumed imported seafood (from Haiti for one and from either Indonesia or Nicaragua for the other). The PFGE pattern combinations of nine $(10 \%)$ serogroup O1 isolates from patients with domestically acquired cholera associated with exposure to seafood from the Gulf Coast, labeled the 'Gulf Coast strain,' were indistinguishable from each other and from the established pattern of the Gulf Coast strain [23]. Of the remaining 39 serogroup O1 isolates, labelled 'Other patterns', 23 reported travel to South Asia [nine $(39 \%)$ each to India and Pakistan, four $(17 \%)$ to Bangladesh, and one (4\%) to Nepal]; eight to Southeast Asia [five $(63 \%)$ to the Philippines) and three (38\%) to Indonesia]; five reported no travel; and one had unknown exposure, but was a resident of Guam. The PFGE pattern of the serogroup O139 isolate was similar but not identical to PFGE patterns of serogroup O1 and O139 isolates from patients who had travelled to countries in Southeast Asia.

Antimicrobial susceptibility testing was conducted on all $107 \mathrm{~V}$. cholerae $\mathrm{O} 1$ isolates and defined three major antibiotic susceptibility pattern complexes (Table 2). The first is a pan-susceptibility pattern, in which isolates were susceptible to all antimicrobial agents tested. This pattern was seen only in the 10 isolates from patients whose source of infection was Gulf Coast seafood. The second is a complex of multi-drug resistance (MDR) patterns, in which isolates were susceptible to agents from at least five CLSI classes. These MDR patterns were seen in isolates from travellers to Hispaniola $(n=37)$, South Asia $(n=35)$, Africa $(n=2)$, and in several domestically acquired cases that were not linked to Gulf Coast Seafood $(n=3)$. All were resistant to nalidixic acid, and all but four (two from travellers to Bangladesh in 2007 and 
Table 2. Laboratory characterization on isolates of $\mathrm{V}$. cholerae O1, including serogroup/biotype, PFGE pattern, and antimicrobial resistance pattern, United States, 2001-2011 $(n=107)$

\begin{tabular}{|c|c|c|c|c|c|c|c|c|c|c|}
\hline \multirow[b]{2}{*}{ Exposures (source of illness) } & \multirow[b]{2}{*}{$\begin{array}{l}V . \text { cholerae } \mathrm{O} 1 \\
(n=107)\end{array}$} & \multicolumn{2}{|c|}{ Serotype/biotype } & \multicolumn{4}{|c|}{ PFGE pattern } & \multicolumn{3}{|l|}{ Resistance pattern } \\
\hline & & $\begin{array}{l}\text { Inaba } \\
\text { El Tor } \\
(n=22)\end{array}$ & $\begin{array}{l}\text { Ogawa } \\
\text { El Tor } \\
(n=85)\end{array}$ & $\begin{array}{l}\text { 'Haiti' } \\
\text { pattern } \\
(n=45)\end{array}$ & $\begin{array}{l}\text { 'Gulf } \\
\text { Coast' } \\
\text { pattern } \\
(n=9)\end{array}$ & $\begin{array}{l}\text { Other } \\
\text { patterns } \\
(n=39)\end{array}$ & $\begin{array}{l}\text { Not } \\
\text { tested } \\
(n=14)\end{array}$ & $\begin{array}{l}\text { Pan-susceptible* } \\
(n=10)\end{array}$ & $\begin{array}{l}\text { Furazolidone } \\
\text { resistance } \dagger \\
(n=20)\end{array}$ & $\begin{array}{l}\text { Multidrug } \\
\text { resistance } \\
\text { patterns } \\
(n=77)\end{array}$ \\
\hline \multicolumn{11}{|l|}{ Travel-associated cases: destination } \\
\hline Travel: Hispaniola & 37 & 0 & 37 & 36 & 0 & 1 & 0 & 0 & 0 & 37 \\
\hline Travel: South Asia & 35 & 10 & 22 & 6 & 0 & 23 & 6 & 0 & 0 & 35 \\
\hline Travel: Southeast Asia & 13 & 2 & 14 & 0 & 0 & 8 & 5 & 0 & 13 & 0 \\
\hline Travel: Africa & 2 & 0 & 2 & 1 & 0 & 1 & 0 & 0 & 0 & 2 \\
\hline \multicolumn{11}{|l|}{ Domestically-acquired cases } \\
\hline $\begin{array}{l}\text { Gulf Coast seafood } \\
\text { consumption }\end{array}$ & 10 & 10 & 0 & 0 & 9 & 1 & 0 & 10 & 0 & 0 \\
\hline Imported seafood consumption & 3 & 0 & 3 & 2 & 0 & 0 & 1 & 0 & 1 & 2 \\
\hline Other seafood consumption & 5 & 0 & 5 & 0 & 0 & 3 & 2 & 0 & 5 & 0 \\
\hline No seafood consumption & 1 & 0 & 1 & 0 & 0 & 1 & 0 & 0 & 0 & 1 \\
\hline \multicolumn{11}{|l|}{ Unknown exposure } \\
\hline Unknown & 1 & 0 & 1 & 0 & 0 & 1 & 0 & 0 & 1 & 0 \\
\hline
\end{tabular}

PFGE, Pulsed-field gel electrophoresis.

* Pan-susceptible: susceptible to all antimicrobial agents tested.

$\dagger$ Furazolidone resistance: resistant to furazolidone (one isolate also was resistant to nalidixic acid).

$\$$ Multidrug resistance patterns: isolates were resistant to antimicrobial agents from at least five CLSI classes. All of these isolates were resistant to nalidixic acid, streptomycin, and trimethoprim-sulfamethoxazole as well as one or more of the following:tetracycline (four isolates), furazolidone, and sulfamethoxazole. 
2011, one traveller to India in 2008, and one traveller to Pakistan in 2010) were susceptible to tetracycline. The third pattern complex included isolates resistant to furazolidone alone (one was also resistant to nalidixic acid); it was seen in the 13 isolates from travellers to Southeast Asia as well as in seven domestically acquired cases that were not linked to Gulf Coast seafood. Antimicrobial susceptibility testing was also conducted on the isolate of $V$. cholerae $\mathrm{O} 139$; it was resistant to nalidixic acid, streptomycin, sulfisoxazole and trimethoprim-sulfamethoxazole, but was susceptible to furazolidone and the other agents in the panel.

\section{DISCUSSION}

Our review of cholera in the United States from 2001 to 2011 confirms the truth of the saying that even rare and apparently exotic infectious diseases are just an airplane ride away. Less than a month after cholera was first noted in Haiti in October 2010, cases associated with that outbreak had been diagnosed in the United States [24, 25]. In 2011, more than twice as many US cases of cholera were associated with travel to Haiti than had been reported from all sources in any year over the previous decade, reminiscent of the pattern seen in the early 1990s with the outbreaks of cholera in Latin America [8]. Cholera has now become endemic in Haiti and has been imported from Hispaniola to Canada, Spain, Venezuela, and possibly Cuba [21, 26]. Endemic transmission of cholera persists in the Dominican Republic and Cuba, as in Haiti, and poses a continued threat of travelassociated cases, particularly to the United States and the rest of the Western hemisphere.

Travel to Asia and consumption of raw or undercooked seafood continue to be sources of cholera in the United States [7-9]. Cases associated with travel to other cholera-affected countries, primarily in Asia, were reported throughout the review period, with no sign of decrease. The incubation period of cholera is short - typically $12 \mathrm{~h}$ to 2 days - so it is likely that additional cases occur while travellers are abroad and are not captured by this surveillance system. Long travel times from Africa and Asia may mean that cases in travellers to these areas are especially likely not to be diagnosed in the United States. Although sanitation standards in the United States make sustained transmission unlikely, cholera and other waterborne diseases are likely to continue to be imported until safe water and adequate sanitation are available to all worldwide. Several cases were associated with consumption in the United States of raw or undercooked seafood, including not only seafood harvested from the Gulf Coast, a focus of $V$. cholerae $\mathrm{O} 1$ first described in the late 1970s [10], but also imported seafood.

Different states have had markedly different experiences with cholera. Most cases associated with travel to Hispaniola were reported from just two states, New York and Florida. As these are the states with the largest populations of Haitians and Dominicans Florida (251963, 46\%), New York (135836, 25\%)this pattern is not surprising, but it is a reminder of the need for culturally and linguistically appropriate medical care and public health response [27]. In Florida, for example, the state health department produced educational materials on cholera in Haitian Creole for patients and their contacts that greatly facilitated response. Cholera cases associated with travel to other countries, by contrast, were reported by 22 states, with no more than eight cases from any state. Most of these patients had travelled to South Asia or Southeast Asia; only two cases were associated with travel to Africa, although many African countries have been hit hard by cholera in recent years $[28,29]$. The relative lack of US cases associated with travel to Africa likely reflects, at least in part, low numbers of travellers, relative to other destinations. A pattern of relatively low numbers of cases - but high risk per traveller - has been reported for other enteric infections [30]. Domestically acquired cholera predominantly affected the Gulf Coast states of Louisiana and Texas, as in years past. In sum, healthcare providers and public health authorities in all states should be prepared to diagnose, treat, and respond to cases of cholera.

Strategies for prevention of cholera in US residents depend to a great extent on the exposure scenario. Of patients with travel-associated cholera, $62 \%$ travelled to visit friends or relatives (VFR) in another country. These types of travellers are less likely than others to seek medical consultation before travel and may also perceive less risk from food and water while travelling $[30,31]$; they can be hard to reach with prevention messages. Other travellers reported travelling for tourism, business, or medical missions or other relief work. Notably, since their risk of exposure may be particularly high, these types of travellers are relatively likely to receive pre-travel medical consultation, which can stress the importance of safe water and food in preventing not only cholera, but many other enteric infections as well. There are currently two 
WHO-prequalified vaccines available outside the United States. Although no cholera vaccines are currently available in the United States, a cholera vaccine intended for US travellers is in development [32]; its use would require a pre-travel healthcare visit. Although we have no data on the proportion of patients who sought pre-travel care, among those for whom information on reason for travel was available and who were not residents of another country, only $24 \%$ were not travelling to visit friends or relatives. For a vaccine to have optimal impact, strategies to reach VFR travelers would be needed. Regarding the potential impact of a vaccine for travellers, it is important to note that US surveillance captures only cases diagnosed in the United States. Finally, prevention of domestically acquired cholera lies squarely in the realm of food safety; the great majority of cases are associated with consumption of raw or undercooked seafood.

Cholera has a well-deserved reputation as a severe, often fatal disease, but prompt and appropriate therapy can all but eliminate fatalities. In our review, $98 \%$ of patients reported diarrhoea; $62 \%$ of those with information on hospitalization were hospitalized. Although none died, several developed severe complications including acute renal failure, cardiopulmonary arrest, and shock. These severe complications from profound dehydration speak to the need for clinicians to be aware of cholera and prepared to treat it appropriately [33, 34]. Importantly, because dehydration progresses rapidly after onset of diarrhoea, patients must be able to access care within a few hours of symptom onset. If a traveller is en route or far from healthcare, cholera gravis can lead to death before a patient reaches a treatment facility. CDC has advised that pre-packaged oral rehydration salts be carried on international flights to address this need [35]. Treatment with antimicrobial agents is adjunctive but can shorten the duration of symptoms and $V$. cholerae shedding [36]. Several public health organizations recommend doxycycline (an antimicrobial in the tetracycline class) as first-line therapy and ciprofloxacin (an antimicrobial in the quinolone class; resistance to nalidixic acid correlates with decreased susceptibility to ciprofloxacin) as an alternative. In our review, isolates from patients whose source of cholera was travel to Southeast Asia or Gulf Coast seafood were susceptible to these agents (except for one isolate from a patient with domestically acquired cholera who ate seafood of unknown source, which was resistant to nalidixic acid). Isolates from patients who had travelled to
Hispaniola, South Asia, and Africa, as well as some who acquired cholera domestically from sources other than Gulf Coast seafood were uniformly resistant to nalidixic acid, but most were susceptible to tetracycline. Within sources of exposure, these resistance patterns did not vary over the period of our review. Macrolide antibiotics are also recommended by some organizations as an alternative agent, but macrolides are not included in the panel of agents tested.

Healthcare providers in the United States should consider cholera in patients, especially adults, with severe watery diarrhoea. The level of suspicion should be heightened for patients with a history of international travel to cholera-endemic regions or of consuming raw or undercooked seafood from the Gulf Coast or elsewhere. Cholera is most often diagnosed by stool culture on thiosulfate citrate bile salts sucrose (TCBS) medium, which in most settings must be specifically requested by the clinician. State and local public health laboratories should send all $V$. cholerae isolates to CDC for confirmatory testing and subtyping by PFGE or, as they become available, newer methods such as whole genome sequencing. CDC can also measure vibriocidal and anti-cholera toxin antibodies in serum.

As with other notifiable infections, surveillance data on cholera in the United States is limited both by under-diagnosis and by under-reporting. Patients may not seek medical care for mild cases, and healthcare providers may not make a specific diagnosis even in severe cases. Reporting by providers and clinical laboratories to states is passive and by state health departments to COVIS is both passive and voluntary. However, under-reporting per se is unlikely to be a major source of bias; a recent comparison of COVIS data to FoodNet active surveillance data showed similar patterns of incidence and trends in Vibrio infections in both systems. Nonetheless, our data should be viewed as a minimal estimate of cholera in the United States.

In summary, this review of US cholera early in the 21st century describes another important chapter in the history of cholera in the United States. This history includes, in the late 1970s, the discovery of the Gulf Coast strain of $V$. cholerae O1 [10]; in the early 1990s, a surge of cases associated with epidemic cholera in Latin America [37-39]; in the late 1990s and early 2000s, a relative lull during which most cases were associated with travel to Asia [7, 8]. Now, in the second decade of the new millennium, we are witnessing a marked increase in cases associated with the 
new epidemic in Hispaniola. Throughout, prevention has depended on basic standards of safe water, sanitation, and food safety. To the extent that the United States can maintain these standards at home and foster their development abroad, it can both protect its own population and contribute to efforts to improve public health in other countries [4].

\section{ACKNOWLEDGEMENTS}

The authors acknowledge the following individuals and groups for their contributions to this work: Patricia Yu, Kelly Jackson, Robert Tauxe, Patricia Griffin, Maurice Curtis, Jessica Halpin, the PulseNet PFGE Laboratory, state and local public health departments.

The findings and conclusions in this report are those of the authors and do not necessarily represent the official position of the Centers for Disease Control and Prevention.

Much of the material in this paper was presented at the 2012 Infectious Diseases Week Conference in San Diego, CA. A small subset of the cases, the first 23 cases diagnosed in the United States associated with the epidemic in Haiti and the Dominican Republic, was published in Emerging Infectious Diseases in 2011. This paper is referenced in our manuscript (Newton et al. [25]); it includes about $20 \%$ of the cases and $<5 \%$ of the time on which we report.

\section{DECLARATION OF INTEREST}

None.

\section{REFERENCES}

1. CDC. Update: cholera outbreak-Haiti, 2010. Morbidity Mortality Weekly Report 2010; 59: 1473-1479.

2. Barzilay EJ, et al. Cholera surveillance during the Haiti epidemic - the first 2 years. New England Journal of Medicine 2013; 368: 599-609.

3. Markel H. A piece of my mind. Happy birthday, Dr Snow. Journal of the American Medical Association 2013; 309: 995-996.

4. Waldman RJ, Mintz ED, Papowitz HE. The cure for cholera -improving access to safe water and sanitation. New England Journal of Medicine 2013; 368: 592-594.

5. Gordon AM. Cholera in the new world. Journal of the Kentucky Medical Association 1970; 68: 657-658.

6. Weissman JB, et al. A case of cholera in Texas, 1973. American Journal of Epidemiology 1974; 100: 487-498.

7. Steinberg EB, et al. Cholera in the United States, 1995-2000: trends at the end of the twentieth century. Journal of Infectious Diseases 2001; 184: 799_ 802.

8. Mahon BE, et al. Reported cholera in the United States, 1992-1994: a reflection of global changes in cholera epidemiology. Journal of the American Medical Association 1996; 276: 307-312.

9. Weber JT, et al. Cholera in the United States, 19651991. Risks at home and abroad. Archives of Internal Medicine 1994; 154: 551-556.

10. Blake PA, et al. Cholera - a possible endemic focus in the United States. New England Journal of Medicine 1980; 302: 305-309.

11. Johnston JM, et al. Cholera on a Gulf Coast oil rig. New England Journal of Medicine 1983; 309: 523-526.

12. Lowry PW, et al. Cholera in Louisiana: widening spectrum of seafood vehicles. Archives of Internal Medicine 1989; 149: 2079-2084.

13. Newton A, et al. Increasing rates of vibriosis in the United States, 1996-2010: review of surveillance data from 2 systems. Clinical Infectious Diseases 2012; 54 (Suppl. 5): S391-395.

14. Fields PI, et al. Use of polymerase chain reaction for detection of toxigenic Vibrio cholerae $\mathrm{O} 1$ strains from the Latin American cholera epidemic. Journal of Clinical Microbiology 1992; 30: 2118-2121.

15. Keasler SP, Hall RH. Detecting and biotyping Vibrio cholerae $\mathrm{O} 1$ with multiplex polymerase chain reaction. Lancet 1993; 341: 1661.

16. Ghosh C, et al. A search for cholera toxin (CT), toxin coregulated pilus (TCP), the regulatory element ToxR and other virulence factors in non-01/non-0139 Vibrio cholerae. Microbial Pathogenesis 1997; 22: 199-208.

17. Nandi B, et al. Rapid method for species-specific identification of Vibrio cholerae using primers targeted to the gene of outer membrane protein OmpW. Journal of Clinical Microbiology 2000; 38: 4145-4151.

18. Cooper KL, et al. Development and validation of a PulseNet standardized pulsed-field gel electrophoresis protocol for subtyping of Vibrio cholerae. Foodborne Pathogens and Disease 2006; 3: 51-58.

19. Clinical and Laboratory Standards Institute (CLSI). Methods for antimicrobial dilution and disk susceptibility testing of infrequently isolated or fastidious bacteria; approved guidelines. Wayne, PA: CLSI, 2010.

20. Clinical and Laboratory Standards Institute (CLSI). Performance standards for antimicrobial susceptibility testing; twentieth informational supplement. Wayne, PA: CLSI, 2010.

21. Jimenez ML, et al. Multinational cholera outbreak after wedding in the Dominican Republic. Emerging Infectious Diseases 2011; 17: 2172-2174.

22. Talkington D, et al. Characterization of toxigenic Vibrio cholerae from Haiti, 2010-2011. Emerging Infectious Diseases 2011; 17: 2122-2129.

23. Cameron DN, et al. Molecular characterization of Vibrio cholerae O1 strains by pulsed-field gel electrophoresis. Journal of Clinical Microbiology 1994; 32: 1685-1690.

24. CDC. Update on cholera-Haiti, Dominican Republic, and Florida, 2010. Morbidity Mortality Weekly Report 2010; 59: 1637-1641. 
25. Newton AE, et al. Cholera in United States associated with epidemic in Hispaniola. Emerging Infectious Diseases 2011; 17: 2166-2168.

26. PAHO. Epidemiologic alert: cholera situation update. Epidemiologic Alert, 2012.

27. Bureau UC. 2009 American community survey and Puerto Rico survey 1-year estimates, 2009.

28. WHO. Cholera, 2011. Weekly Epidemiologic Record 2012; 31-31: 289-304.

29. Gaffga NH, Tauxe RV, Mintz ED. Cholera: a new homeland in Africa? American Journal of Tropical Medicine and Hygiene 2007; 77: 705-713.

30. Kendall ME, et al. Travel-associated enteric infections diagnosed after return to the United States, Foodborne Diseases Active Surveillance Network (FoodNet), 2004-2009. Clinical Infectious Diseases 2012; 54 (Suppl. 5): S480-487.

31. Leder $\mathbf{K}$, et al. Illness in travelers visiting friends and relatives: a review of the GeoSentinel Surveillance Network. Clinical Infectious Diseases 2006; 43: 11851193.

32. Chen WH, et al. Safety and immunogenicity of singledose live oral cholera vaccine strain CVD 103-HgR, prepared from new master and working cell banks. Clinical and Vaccine Immunology 2014; 21: 66-73.

33. Besser RE, et al. Diagnosis and treatment of cholera in the United States. Are we prepared? Journal of the American Medical Association 1994; 272: 1203-1205.

34. Eberhart-Phillips J, et al. An outbreak of cholera from food served on an international aircraft. Epidemiology and Infection 1996; 116: 9-13.

35. Brunkard JM, Newton AE, Mintz ED. Cholera. In: The Yellow Book: CDC Health Information for International Travel 2014, 2014, pp. 159.

36. CDC. Antibiotic treatment: recommendations for the use of antibiotics for the treatment of cholera.

37. Ries AA, et al. Cholera in Piura, Peru: a modern urban epidemic. Journal of Infectious Diseases 1992; 166: 1429-1433.

38. Tauxe RV, Mintz ED, Quick RE. Epidemic cholera in the new world: translating field epidemiology into new prevention strategies. Emerging Infectious Diseases 1995; 1: 141-146.

39. Pan American Health Organization, WHO. Update: the cholera situation in the Americas. Epidemiological Bulletin 1991; 12: 11-12. 